\title{
A study of gas bubbles in liquid mercury in a vertical Hele-Shaw cell
}

\author{
B. Klaasen ${ }^{1, \star}$, F. Verhaeghe ${ }^{1,2}$, B. Blanpain ${ }^{1}$, J. Fransaer $^{3}$
}

${ }^{1}$ Research group for High Temperature Processes and Industrial Ecology, Department of Metallurgy and Materials Engineering, KU Leuven, Kasteelpark Arenberg 44, 3001 Leuven, Belgium

${ }^{2}$ Umicore Group Research \& Development, Watertorenstraat 33, 2250 Olen, Belgium

${ }^{3}$ Research group for Materials with Novel Functionality, Department of Metallurgy and Materials Engineering, KU Leuven, Kasteelpark Arenberg 44, 3001 Leuven, Belgium

* corresponding author, email: mail@bartklaasen.be

\section{ABSTRACT}

High quality observations of mesoscopic gas bubbles in liquid metal are vital for a further development of pyrometallurgical gas injection reactors. The opacity of metals however enforces the use of indirect imaging techniques with limited temporal or spatial resolution. In addition, accurate interface tracking requires tomography which further complicates the design of a high temperature experimental setup. In this paper an alternative approach is suggested that circumvents these two main restrictions. By injecting gas in a thin layer of liquid metal entrapped between two flat and closely spaced plates, bubbles in a Hele-Shaw flow regime are generated. The resulting quasi-2D multiphase flow phenomena can be fully captured from a single point of view and, when using a non-wetted transparent plate material, the bubbles can be observed directly. The feasibility of this approach is demonstrated by observations on buoyancy driven nitrogen bubbles in liquid mercury in a vertical Hele-Shaw cell. By using a moving high speed camera to make continuous close up recordings of individual bubbles, the position and geometry of these bubbles are quantified with a high resolution along their entire path. After a thorough evaluation of the experimental accuracy, this information is used for a detailed analysis of the bubble expansion along the path. While the observed bubble growth is mainly caused by the hydrostatic pressure gradient, a careful assessment of the volume variations for smaller bubbles shows that an accurate bubble description should account for significant dynamic pressure variations that seem to be largely regime dependent.

This text was published in EXPERIMENTS IN FLUIDS:

B. KlaAsen, F. Verhaeghe, B. Blanpain, J. Fransaer, Exp Fluids, vol. 55:1652, 2014

The final publication is available at www.springerlink.com:

http://dx.doi.org/10.1007/s00348-013-1652-x 


\section{INTRODUCTION}

Many industrial pyrometallurgical processes rely on bubble column reactors in which gas bubbles are blown directly into a metal melt through tuyeres or immersed lances (Engh et al. 1992). The result is intensive mixing and a large contact area between the gas phase and the liquid metal, despite the relatively compact reactor design. For this reason it is expected that the on-going trend towards process intensification will go hand in hand with an increased use of gas injection reactors in pyrometallurgy (Grechko 2000). Yet, this requires significant research efforts to ensure that the understanding of the governing mechanisms grows simultaneously. Such research has to adopt a multiscale approach because the relevant phenomena occur on time and length scales spanning up to six orders of magnitude (Bauer and Eigenberger 2001). On the largest scale flow patterns, average phase distributions and mass balances of reactors are described, while on the smallest scales physicochemical reactions are studied. Often the highly complex interactions between these macroscopic and microscopic phenomena determine the process characteristics to a large extent. Nevertheless, a limited understanding of these mesoscopic interactions is probably the most important bottleneck when designing and optimizing reactors. This can be solved by investigating individual gas bubbles, whose properties are directly affected by these interactions.

A possible way to study gas bubbles is through simulation. Numerous examples of mainly CFD based simulations of bubble column reactors exist. Good overviews are provided by Iguchi and llegbusi (2011) for macroscopic models of metallurgical applications and by Jakobsen et al. (2005) for mesoscopic bubbles in general. Yet most of these simulations rely indirectly on experimental results incorporated through closure laws that account for small scale effects which are not directly resolved by the simulation. This means that detailed experimental observations are indispensable for the development and validation of mesoscopic bubble models. Hence, experimental efforts should not be abandoned (Jakobsen et al. 2005).

Experimental observations of gas bubbles in liquid metal are hindered by the opaqueness of the bulk liquid, preventing a direct visualization of bubbles. A second difficulty is the high melting point of most metals; the fact that observations often need to be made at elevated temperatures complicates the design of an experimental setup. So while a wide range of techniques exists to observe bubbly flow in low melting transparent media like aqueous or oil based solutions (Boyer et al. 2002), possibilities in liquid metals are limited. When investigating macroscopic flow properties in reactors, these limitations are often circumvented by extrapolating observations in water models to liquid metal conditions (Mazumdar and Evans 2004). Yet, while macroscopic predictions can be compared against observations in real metal melts by comparing process parameters like bubbling frequency, splash height and plume eye dimensions, it is much less clear how possible mesoscopic observations can be verified when their effect is not known a priori. The question of validation is especially important when dealing with reactive systems, as mass transfer can profoundly affect the bubble regime (Taylor et al. 1996). Hence, to study reactive gas injection in liquid metals with physical models, translucent systems with comparable hydrodynamic and thermokinetic properties are required. Unfortunately these are rarely available. 
To summarize, the authors are convinced that research on gas injection in pyrometallurgy should primarily focus on the behavior of individual bubbles at a mesoscopic scale. While simulation techniques can definitely support such research, currently experimental observations are indispensable to make significant progress in this field. Moreover, considering the large difference in physicochemical properties between water and metals, such observations should be carried out in actual metallic melts.

\subsection{AVAILABLE TECHNIQUES}

A first way to observe bubbles in liquid metals is by using invasive probes that detect locally whether gas or liquid is present. Various designs exist, including electrical resistivity probes (Oryall and Brimacombe 1976), electrical capacitance probes (Fu and Evans 1999) and acoustic probes (Schneider and Evans 2006). An inherent disadvantage of this technique is however the disturbance of the flow due to the interaction with the sensors. In addition, the spatial resolution of the observations is limited even when arrays of probes are used (Iguchi et al. 1997). So while the results can be used to estimate bubble distributions and overall bubble shapes, an accurate reconstruction of the bubble interface, required for studying mesoscopic interactions, is not possible.

Alternatively, tomographic techniques can be used to map phase distributions through differences in physical properties measured by non-invasive sensors. Based on the interaction between the fluids and the interrogating signals, a distinction between soft field and hard field tomography is made (Marashdeh et al. 2008). In the first case, the shape of the field lines of the interrogating signals is affected by the physical property distribution. For metals the authors found examples on the use of acoustic (Schwerdtfeger 1968; Munshi et al. 1998; Zhang et al. 2007), electrical (Wang et al. 2010) or magnetic signals (Terzija et al. 2011). Yet the results are limited to average flow descriptions in tubular geometries, as the spatial resolution is insufficient to provide details on instant bubble shapes. This is a common limitation of soft field tomographic techniques, for which the reconstruction of fluid properties is inherently more complex and less sensitive compared to hard field tomography based on radiographic imaging. Therefore, the use of the latter seems to be more appropriate. In the past, bubbles in metals were visualized using X-rays (Heindel 2011; Baker and Bonazza 1998; Iguchi et al. 1995), neutron beams (Saito et al. 2005; Saito et al. 2009) and $\gamma$-rays (Thiyagarajan et al. 1995). Because the field lines of these signals are not bent, spatial property mapping is significantly more accurate. A drawback is however the limited penetration depth in metallic systems, restricting the use of this technique to geometries with limited cross section. In addition, the use of high intensity sources requires a complete and secure shielding of the setup. Especially in combination with heating systems and thermal insulation, this limits the possibilities of making simultaneous observations from multiple angles required for tomographic reconstruction. So despite that hard field tomography is the most promising technique to study mesoscopic bubbles with high resolution, currently only $2 \mathrm{D}$ radiography has been applied onto liquid metal systems, averaging out the third dimension. 


\subsection{A QUASI-2D APPROACH}

Instead of trying to overcome the experimental limitations inherent to the application of 2D imaging techniques for mapping 3D flow patterns, in this paper the possibility of studying bubbles in liquid metal flows under quasi-2D circumstances is explored. By entrapping a thin sheet of liquid metal between two flat parallel plates, a Hele-Shaw flow regime is approached: Stokes flow between two parallel plates at infinitesimal distance. After injection of bubbles in such a so-called Hele-Shaw cell, flow phenomena develop as in figure 1. While Hele-Shaw flows in theory develop only in two dimensions, in practice the non-zero cell thickness will result in a non-uniform flow profile over the cell gap, perpendicular to the plane of the cell. This profile however, can be calculated to be parabolic when laminar flow and non-slip boundary conditions on the walls are assumed (Vasil'ev 2009). For most flows in cells with limited thickness these assumptions are valid because drag forces exerted by the sidewalls slow down the fluid, suppress turbulence and stabilize laminar flow patterns (Beavers et al. 1970). Consequently, the fluid velocity at any point in the cell can be calculated from a description of the average flow patterns in the plane of the cell. Therefore flows in Hele-Shaw cells can be treated as being quasi-2D: despite their three dimensional character, they can be fully captured by two dimensional observations from a single point outside the plane, avoiding the need to apply a tomographic reconstruction.

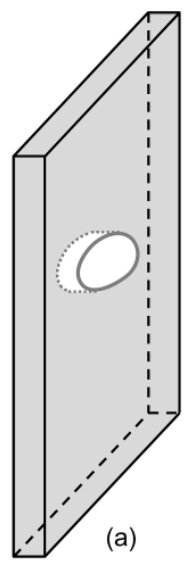

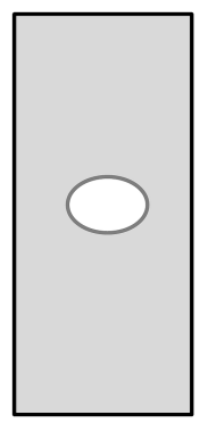

(b)

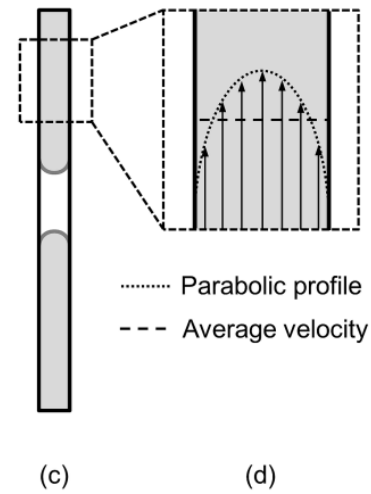

Fig. 1 Illustration of a Hele-Shaw cell containing liquid and a gas bubble. When the volume of the bubble in a HeleShaw cell is sufficiently large, it is squeezed between the sidewall and adopts a disc shape. (a), (b) and (c) show this in isometric, front and side view. (d) shows a close up of the parabolic flow profile over the gap between the walls, together with the average velocity.

Despite the additional boundary conditions imposed by the sidewalls, most phenomena that govern bubbly flows in bulk liquids are also relevant for flows in Hele-Shaw cells. Hence, the same mesoscopic interactions can be studied. Examples could be the effect of mass transfer on bubble hydrodynamics, the distribution of reaction products over the phases, the temperature distribution in the fluids, the influence of surfactants, the effect of fluid properties etc. Such specific knowledge can be used to validate simulations for Hele-Shaw conditions directly, or translated into closure laws for modeling other geometries. Clearly this would often require the application of these closure laws outside their validated range, as the relative importance of different phenomena under Hele-Shaw conditions can be different compared to other geometries. It is for example clear that the volume to surface ratio of bubbles under Hele-Shaw conditions can differ significantly from that of bubbles in the bulk of 
a liquid. Because a validation of such extrapolations can only come from three dimensional experiments, a quasi-2D approach will never be able to replace 3D observations completely. Nevertheless the authors believe that its simplicity could make it well-suited for an initial and fast exploration of the mesoscopic behavior of bubbles in liquid metals. Yet first it should be verified that detailed and relevant observations on bubbles in metals in a Hele-Shaw cell are feasible. Hence, the main goal of this work is to explore the possibilities and limitations of the suggested approach.

The remainder of this paper is organized as follows. First, to conclude this introduction, an overview of earlier work on bubbly flows in Hele-Shaw cells is presented. Next, in section 2, the experimental setup is introduced. In section 3 a set of observations is presented and discussed with a focus on the evolution of the bubble size. It is the starting point for an evaluation of the accuracy of the experimental approach in section 4.1. Afterwards, in 4.2, an explanation for observed irregularities in the bubble growth is suggested.

Properties of bubbles in various gas-liquid combinations have already been studied under Hele-Shaw conditions. Older publications describe experiments in cells that are positioned horizontally or tilted to a limited inclination with the horizontal. Results are reported for air bubbles in water (Johann and Siekmann 1978), oil (Maxworthy 1986; Park et al. 1994) and aqueous alcohol solutions with variable viscosity (Eck and Siekmann 1978; Kopf-Sill and Homsy 1988). These low Reynolds number flows are well-suited to simulate microgravity conditions and to validate analytical flow descriptions. For pyrometallurgical applications however, vertically rising, buoyancy driven bubbles are more relevant. Such scenarios were only studied more recently by Kelley and Wu (1997), Bush (1997), Bush and Eames (1998), Roig et al. (2012), Huisman et al. (2012) and Bouche et al. (2012) for air bubbles rising in water, and by Kawaguchi et al. (2006), Kozuka et al. (2009) and Yamamoto and Kawaguchi (2011) who also considered aqueous polymer solutions. Finally the work of He et al. (2011) on bubbles rising in aqueous suspensions of magnetic particles can also be mentioned. In all cases, bubbles are observed directly through transparent cell walls, so no radiography is applied. This means that all investigated liquids had to be optically transparent. The reason is that aqueous solutions tend to have a high wettability on most cell materials, causing the formation of a thin liquid boundary layer on the cell surface, shielding the gas from the sidewalls. So only when this film is transparent, bubbles can be observed directly. It is probably the reason why an extensive search in the open literature did not yield any examples of experiments on gas injection in opaque liquids in Hele-Shaw cells. Observations on bubbles in opaque liquid metal require the use of radiography unless a transparent cell material can be found that is not wetted by the metal. Fortunately, liquid metals typically have a large surface tension and do not tend to wet surfaces, at least if no chemical bonding with the surface material occurs. Hence it should be possible to observe bubbles in opaque liquid metal directly through a Hele-Shaw cell if the latter can be constructed from a compatible transparent material able to withstand temperatures above the melting point of the metal. This has been partially confirmed by the work of Paneni and Davenport (1969), who indeed observed bubbles in liquid mercury through a transparent glass cell. Similar to a Hele-Shaw cell, their crucible also has a rectangular cross section, but the gap of $4.7 \mathrm{~mm}$ between the walls is too large to establish a Hele-Shaw flow profile. As a result, the bubbles only touch one side of the cell, leaving the other side covered with an inhomogeneous layer of mercury. 


\section{EXPERIMENTAL SETUP}

Considering the exploratory goal of the current work, difficulties related to the practical construction of a setup are avoided as much as possible. Firstly this is done by selecting a very simple system: buoyancy driven bubbles of inert nitrogen gas rising in liquid mercury. The low melting point of mercury $(234 \mathrm{~K})$ allows isothermal experiments at room temperature (297 K) without applying additional heating. Secondly, a Hele-Shaw of optically transparent soda-lime glass is used. The experiments of Paneni and Davenport (1969) show that this material is poorly wetted by mercury, so that the bubbles can be observed directly through the glass and recorded by an optical camera. Figure 2 shows the setup used in this work.

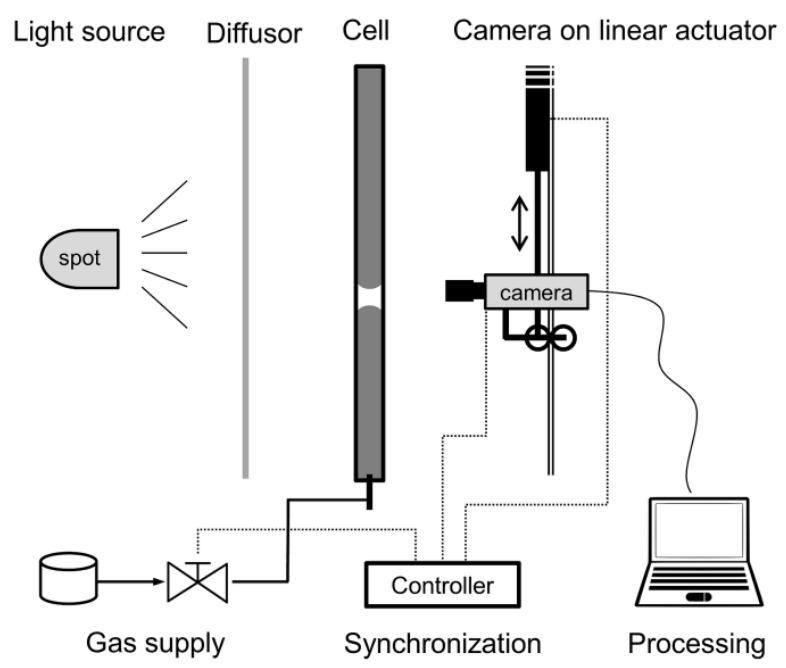

Fig. 2: The setup, consisting of a Hele-Shaw cell aligned with a diffuse light source on the one side and a high-speed camera on the other side. This camera is mounted on a vertical rail and positioned by a hydraulic actuator. Gas is supplied in bursts through a needle at the bottom of the cell. The length of the burst is controlled by an electromagnetic valve. The opening of this valve is synchronized with the onset of the camera motion and recording. After recording, the captured frames are transferred from the camera to a computer for post processing.

\subsection{Cell CONStRuction}

As shown in figure 1, the flow in a Hele-Shaw cell strongly depends on the distance between the walls. A smaller thickness $t$ results in slower fluid velocities. In the limiting case of zero thickness, all flow is prohibited by wall friction. On the other hand, for larger thicknesses, drag forces will not be able to suppress the development of turbulence, resulting in a disturbance of the quasi-2D flow nature. Based on experiments in thin channels with varying thickness and different irregularities, Beavers et al. (1970) conclude that transitions to turbulent flow occur at Reynolds numbers $\operatorname{Re}=u t / v$ between 2200 and 3400, where $u$ represents the fluid velocity and $v$ the kinematic viscosity of the liquid. Hence, in the previously mentioned studies on bubbles in aqueous solutions $\left(v_{\mathrm{H}_{2} \mathrm{O}}=9.0 \times 10^{-7} \mathrm{~m}^{2} / \mathrm{s}\right)$ with cell thickness ranging from $1.0 \mathrm{~mm}$ (Roig et al. 2012; Huisman et al. 2012; Bouche et al. 2012) to $2.5 \mathrm{~mm}$ (Bush and Eames 1998; Bush 1997) and velocities remaining below 0.2 $\mathrm{m} / \mathrm{s}$, the laminar character of the flow is preserved. However, with mercury as the working liquid, Reynolds numbers for similar cells will be larger due to the smaller viscosity $v_{H g}=1.13$ $x 10^{-7} \mathrm{~m}^{2} / \mathrm{s}$. When assuming that buoyancy driven bubbles in mercury obtain similar velocities as in water, as has been observed by Paneni and Davenport (1969) in their earlier discussed work, this limits the cell thickness to about $1.2 \mathrm{~mm}$ in order to ensure laminar flow conditions. 
Hence in this work a cell thickness of about $1 \mathrm{~mm}$ was selected, allowing for velocities of at least $0.25 \mathrm{~m} / \mathrm{s}$ under laminar flow conditions. This should be sufficient as the observed velocities are indeed similar to those in water.

As mentioned before, the soda-lime glass cell walls are poorly wetted by mercury. In combination with the high surface tension $\left(\sigma_{H g}=0.487 \mathrm{~N} / \mathrm{m}\right)$, this results in a contact angle $\vartheta_{\mathrm{Hg}}$ of about $130^{\circ}$ (Smithwick 1988). Before assembly, two $6 \mathrm{~mm}$ thick rectangular glass plates are cleaned for 20 minutes in piranha solution $\left(3: 1\right.$ mixture of $96 \% \mathrm{H}_{2} \mathrm{SO}_{4}$ and $30 \%$ $\mathrm{H}_{2} \mathrm{O}_{2}$ ), removing organic surface active contaminants and rendering the surface hydrophilic. After rinsing, the heterogeneity of the surface was verified by a visual inspection of the spreading of de-ionized water films on the glass. Then the plates were clamped onto stainless steel spacers, resulting in a cell gap with an average thickness $t$ of $1.049 \mathrm{~mm}( \pm 18$ $\mu \mathrm{m})$. A U-shaped silicone seal placed in this gap creates a channel of $50.0 \mathrm{~mm}( \pm 0.5 \mathrm{~mm})$ width. It is filled up to a height $H$ of $460 \mathrm{~mm}$ above the bottom with liquid mercury $199.99 \%$ purity). Nitrogen gas of technical purity is injected through a vertical needle inserted at the bottom of the cell, with the tip at $10 \mathrm{~mm}$ from the cell bottom. The needle has an inner diameter of $0.5 \mathrm{~mm}$ and a sharp tip to facilitate the detachment of gas bubbles. The gas is injected in bursts from a supply at 1 bar gauge pressure. Burst durations are controlled by an electromagnetic micro-valve (type Burker 2282) steered by a calibrated pulse generator. Considering the small flow rate, long supply channel length and limited pressure difference over the valve, thermal equilibrium between gas and liquid can be assumed the point of injection.

\subsection{IMAGING SYSTEM}

As illustrated in figure 2, one side of the cell is illuminated by indirect lighting through a diffusive screen. The presence of this screen ensures a soft, homogeneous light intensity over the entire cell. It also prevents the formation of temperature gradients over the cell due spatial variations in irradiated heat from the light source. In this configuration, high contrast observations of bright bubbles surrounded by dark opaque liquid metal can be made from the opposite side of the cell. These are recorded with a Tesin Cyclocam high speed camera mounted on a moving platform. Driven by a hydraulic actuator, this platform makes a translation motion in the vertical direction with about the same velocity as that of a rising bubble. Hence, by coordinating this motion with the gas injection, the entire path of the bubbles can be recorded, from the detachment at the needle to the breakup at the top surface. The bubble is thus observed in a Lagrangian reference frame attached to the camera. The corresponding velocity is determined from a pattern of equidistant markers (4 $\mathrm{mm}$ spacing) fixed onto the cell.

Figure 3 shows an example of a single frame as captured by the camera. These 8 bit grayscale frames are recorded with a 480 px by 640 px resolution using a shutter time of 2 $\mathrm{ms}$ and a frame rate of $250 \mathrm{fps}$. Furthermore, by using an 18-108 $\mathrm{mm}$ variable lens and adjusting the optical zoom level, it is ensured that the short side of the image covers exactly the width of the cell, yielding a spatial resolution of $125 \mu \mathrm{m} / \mathrm{px}$. These settings allow an accurate spatial reconstruction of the bubble interface and a detailed tracking of the bubble path over time, while limiting redundancy in the data stream (Versluis 2013). 


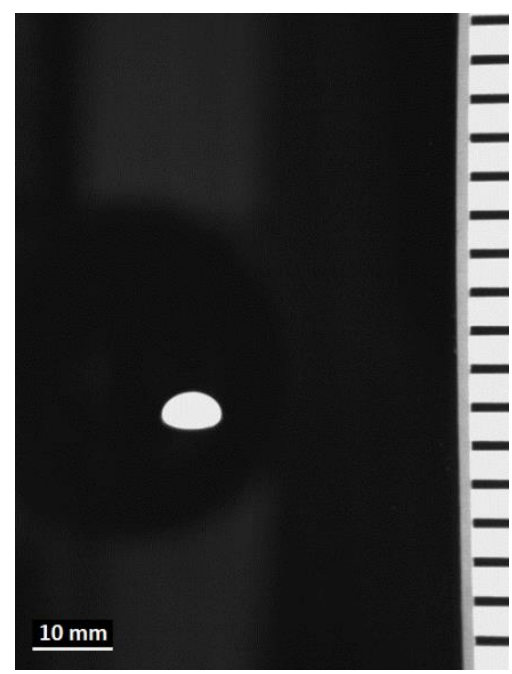

Fig. 3: A typical frame as captured with the camera, before post processing. It shows a bright bubble surrounded by dark liquid metal. The marker pattern on the right is used to determine the position and velocity of the camera.

\subsection{POST PROCESSING}

The image frames are sequentially analyzed with a Matlab (V.2012a) based processing script. First the right section containing the ruler pattern is processed separately. By tracking the relative position change of the markers over consequent frames, the instantaneous camera position and velocity are interpolated as a function of time. Next, the position of the gas-liquid bubble interface is mapped based on the contrast distribution in the left part of the image. This interface is marked by a sharp spatial gradient in contrast between the bubble and the surrounding liquid. During processing, the gas-liquid interface is assumed to be halfway this span. With this information, the properties of the bubble are quantified. In particular the equivalent bubble diameter $d=2 \sqrt{A / \pi}$ is calculated, with $A$ being equal to the area of the region within the bubble interface. Further in the text, this quantity will be used as a measure for the bubble size. Afterwards the position and velocity of the bubble are determined by taking the center of gravity of $A$ as reference point. Here it should be noticed that all descriptions in this paper refer to the absolute position of the bubbles, resulting from a superposition of the camera motion and the position change of the bubble inside the camera's reference frame. Finally the bubble contours can be plotted over time, as shown in figure 4 and figure 8.

\section{OBSERVATIONS}

A key variable in any mesoscopic description is the bubble size, considering that steady state hydrodynamics can be fully mapped as a function of this parameter, the system properties and, in case of confined flow, the channel geometry. Hence, to explore and assess the possibilities and limitations of the current setup, results from an initial study on the quantification and prediction of the bubble size are presented and discussed.

\subsection{FLOW REGIMES}

The range of bubble sizes that can be generated in a Hele-Shaw cell depends on the capillary properties of the system, counteracting the upward buoyancy force. This buoyancy 
over capillarity ratio is expressed by the Eötvos number Eo. Using a characteristic length scale $d / 2$, this number is calculated as Eo $=\Delta \rho g d^{2} /\left(4 \sigma_{H g}\right)$, where $\Delta \rho$ equals the fluid density difference and $g$ the gravitational acceleration $\left(9.81 \mathrm{~m} / \mathrm{s}^{2}\right)$. For very small bubbles (Eo $<1$ ), capillary effects prevent a detachment from the orifice. By supplying additional burst of gas these bubbles can be grown around the injection point. When the Eötvos number approaches 1 , at bubble sizes just under $4 \mathrm{~mm}$ in the current $\mathrm{N}_{2}-\mathrm{Hg}$ system, it is possible to force a detachment of the bubbles by vibrations. These bubbles will rise upward with a low and highly irregular velocity, showing "slip and stick" behavior at random places. Only when buoyancy becomes dominant, for bubbles above $4 \mathrm{~mm}$ diameter, a spontaneous detachment and uninterrupted upward motion is observed. By varying the burst length of the gas supply, single bubbles with variable diameter up to $12 \mathrm{~mm}$ can be injected at once in the current setup. Injection of more gas results in the formation of bubble trains: multiple bubbles that detach consecutively from the needle. These bubble tend to coalesce on their way up due to the nature of the wake (Huisman et al. 2012), eventually forming single bubbles with even larger diameters. In the current setup, this allows the observation of bubbles up to 20 $\mathrm{mm}$ diameter. Larger bubbles could not be created because longer bubble trains did not merge completely before reaching the surface. Table 1 provides a summary.

Table 1: Bubble sizes and corresponding behavior. This study is limited to the $1<$ Eo $<10$ range.

\begin{tabular}{|l|l|l|}
\hline Eo range & $\begin{array}{l}\mathbf{d}(\mathbf{m m}) \text { at } \\
\text { injection }\end{array}$ & Description \\
\hline$<<1$ & $<3$ & No detachment, holdup around orifice \\
\hline$\leq 1$ & $3<4$ & Forced detachment, irregular 'slip and stick' motion \\
\hline $1<10$ & $4<12$ & Injection of single bubbles \\
\hline $10<30$ & $12<20$ & Injection of short bubble trains, coalescence \\
\hline$>30$ & $>20$ & Continuous bubble trains \\
\hline
\end{tabular}

Figure 4 shows a mapping of the bubbles in the Eo range between 1 and 10 . The small bubbles in this range, with a diameter below $5.3 \mathrm{~mm}$, exhibit a linear regime with a constant circular or elliptical shape and a straight path (figure 4-a,b) while bubbles with a diameter over $6.4 \mathrm{~mm}$ show a periodic regime with an oscillating, deformed shape along a swirling trajectory (figure 4-c,d,e,f). In between these regimes bubbles exhibit a mixed behavior, often showing a linear to periodic regime transition somewhere along the path (figure 4-g). The driving force causing this transition is the expansion of the bubble under decreasing hydrostatic pressure along the ascending path. 


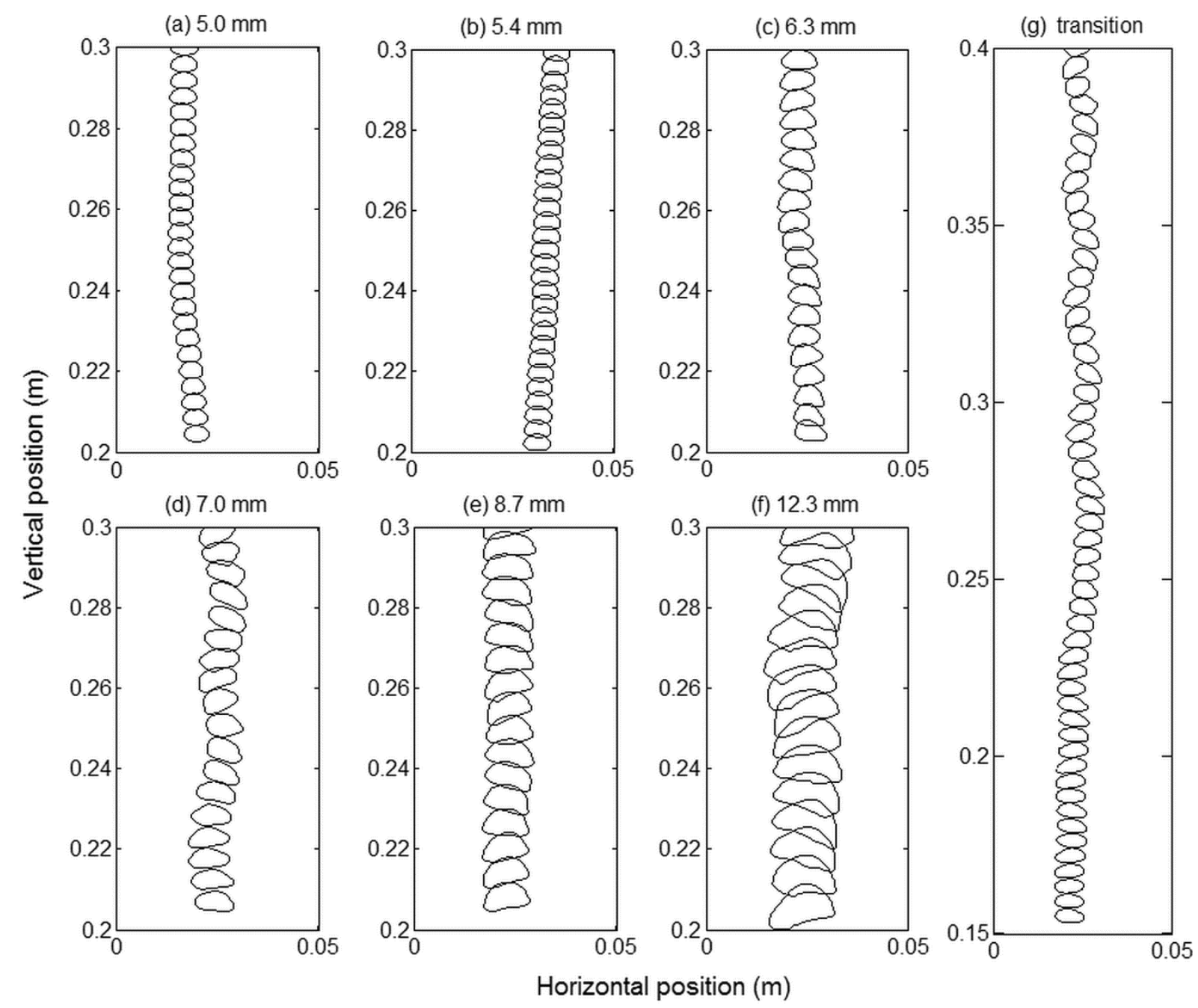

Fig. 4: Successive contour of individual bubbles plotted at a time interval of $0.04 \mathrm{~s}$. The axes define the position of the bubble within the cell $(\mathrm{m})$. The size of the bubbles corresponds to the equivalent diameter of the first plotted bubble. Different sizes are selected that give a good representation of the observed bubble properties. Figures (a-f) show bubbles exhibiting a regular behavior between a position of $20 \mathrm{~cm}$ and $30 \mathrm{~cm}$. Figure (g) shows a bubble of $5.7 \mathrm{~mm}$ changing from a linear regime to a periodic regime along its ascent.

\subsection{BUBBLE GROWTH}

The volume of an inert compressible gas bubble rising upwards in a column of liquid will increase due to a decreasing hydrostatic pressure $P_{\text {hyd }}$ along its trajectory. This hydrostatic pressure can be calculated as a function of the vertical position $y$ in the cell:

$$
P_{h y d}(y)=\rho g(H-y)
$$

In other studies on buoyancy driven bubbles, the hydrostatic expansion between top and bottom is often disregarded, as the resulting effects are small (Santos et al. 2008). When using water as the bulk liquid in the current setup, the maximal pressure difference would be only $4.5 \mathrm{kPa}$. For mercury however, the relatively large density $\left(13.546 \mathrm{~kg} / \mathrm{m}^{3}\right)$ causes a pressure difference of $59.8 \mathrm{kPa}$ between the injection point and the surface of the bath, which is significant compared to the total pressure $P$ in the bubble: 


$$
P(d, y)=P_{a t m}+P_{h y d}(y)+P_{c a p}(d)
$$

where $P_{\text {atm }}$ represents a constant atmospheric pressure $(101.3 \mathrm{kPa})$ and $P_{\text {cap }}(d)$ equals the capillary pressure exerted by the interface as described by Laplace's law. After averaging over the interface, it is calculated as

$$
P_{c a p}(d)=2 \sigma / d
$$

Notice that the capillary effect of the interface curvature over the cell gap in the perpendicular direction is not taken into account. The reason is that, when neglecting the effect of the composition difference between air and nitrogen on $\vartheta_{H g}$, this effect is counteracted by the inverse curvature of the mercury-air interface at the top of the cell. Under the present experimental conditions, the properties of nitrogen are very well approximated by the ideal gas law (Span et al. 2000):

$$
P V=n \mathrm{R} T
$$

where $V$ is the bubble volume, $n$ the amount of gas in the bubble expressed in moles, $T$ the temperature and $\mathrm{R}$ the universal gas constant. Care was taken to ensure isothermal conditions so that the right hand side of expression (4) is constant for a bubble that does not exchange mass with the surrounding liquid. Hence the expansion of an inert bubble along its trajectory can be extrapolated from a reference point at a vertical position $y^{\text {ref }}$ by combining (4) and (2):

$$
V^{I G}(y)=\frac{P_{a t m}+P_{h y d}\left(y^{r e f}\right)+P_{c a p}\left(d^{r e f}\right)}{P_{a t m}+P_{h y d}(y)+P_{c a p}\left(d^{I G}\right)} V\left(d^{r e f}\right)
$$

In this expression, $V^{I G}$ is the predicted volume according to the ideal gas law and $V\left(d^{r e f}\right)$ is the volume of a bubble with equivalent diameter $d^{r e f}$, observed at the reference point. Furthermore, $d^{I G}$ is an equivalent diameter that corresponds to the predicted volume and can be approximated as $\sqrt{4 V^{I G} / t \pi}$. Yet, this relation should be used carefully. Although it is common practice for bubbles in Hele-Shaw flows to calculate the volume $V$ as $t A$, this relation is only valid for the hypothetical case of $2 \mathrm{D}$ flow, when the cell thickness approaches zero and the curvature of the interface over the cell gap can be ignored. However, in order to make a detailed comparison between the extrapolated bubble volume $V^{I G}$ and the actually observed bubble volume $V$, it seems recommended to account for the surface curvature when determining the bubble volume $V$ from the bubble contour surface $A$ or equivalent diameter $d$ as observed by the camera. The capillary length of mercury, calculated as $\sqrt{\sigma / \rho g}$, equals about $1.9 \mathrm{~mm}$. Because this is larger than the distance between the cell walls, the interface will be curved over the entire gap (de Gennes et al. 2003). When assuming that this curvature is uniform, the interface can be described by a circle segment. The endpoints of this segment intercept the cell walls at an angle $\vartheta$ equal to $\vartheta_{H g}$, meaning that the interface is concave from a perspective inside the bubble. This is illustrated in figure 5 . 


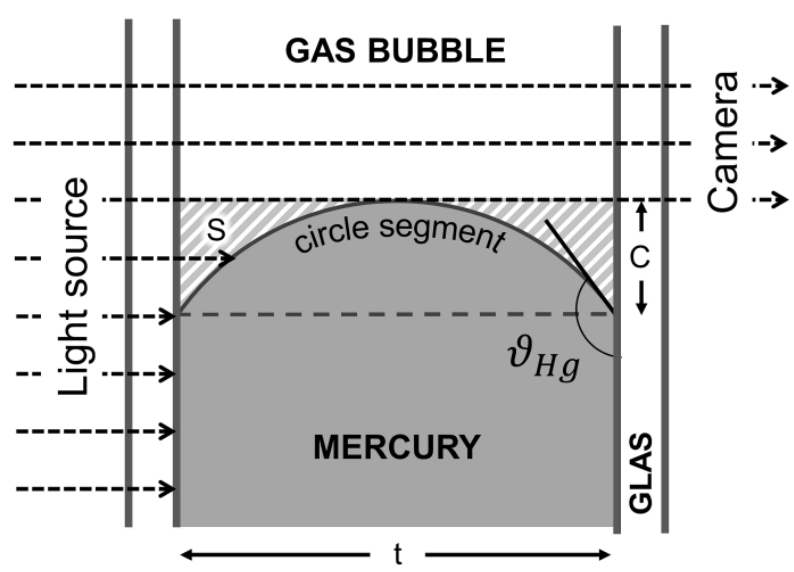

Fig. 5: Cross section of the gas-liquid interface perpendicular to the cell walls.

It is clear that the hatched section contributes to the bubble volume although its projection, with thickness $C$, is not captured by the camera. Hence, based on this description of the interface, it is more accurate to approximate the bubble volume from the observed equivalent diameter $d$ and perimeter $p$ as

$$
V=t \pi d^{2} / 4+p S
$$

with $S$ being the area of the hatched surface. This area can be calculated as the difference between the area covered by the rectangle $t C$ and the area covered by the circle segment, yielding the expression below:

$$
S=\frac{t^{2}}{2}\left(\frac{1-\sin \vartheta}{-\cos \vartheta}\right)-\frac{t^{2}}{8}\left(\frac{2 \vartheta-\pi+\sin 2 \vartheta}{\cos ^{2} \vartheta}\right)
$$

with $\vartheta$ expressed in radians. By combining (6) and (7), it is possible to estimate the bubble volume accurately from the contours observed in the experiments. The results of this calculation are compared against the prediction of the volume expansion by equation (5), using (1), (3) and (4) to calculate the pressure components, for bubbles rising between a reference point taken at $y_{\text {ref }}=15 \mathrm{~cm}$ and a maximal height $y_{\max }$ of $40 \mathrm{~cm}$. Hence, the first and last parts of the path are omitted to exclude transient effects related to the injection or the approach of the upper free surface. In figure 6, the observed and predicted volumes for individual bubbles (depicted in figure 4) are compared. 

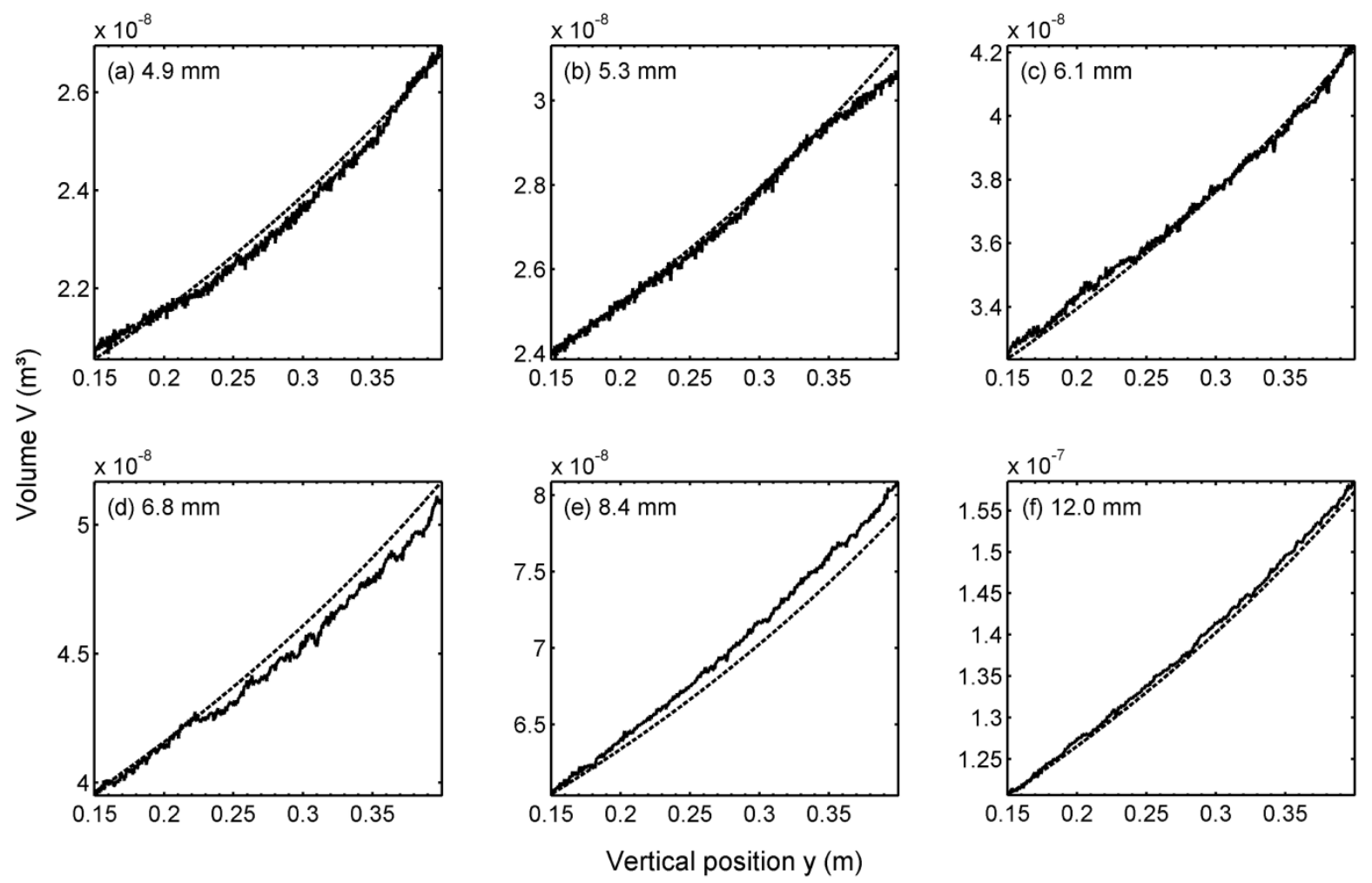

Fig. 6: Measured size (full line) and predicted size (dashed line) as function of the vertical position for the bubbles shown in figure 4.

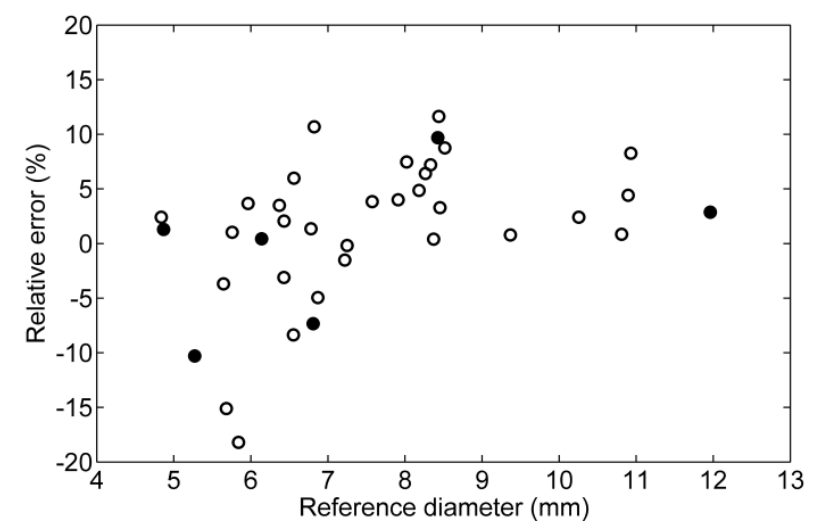

Fig. 7: The deviation between the observed and predicted bubble growth over the path section between $y^{r e f}=15 \mathrm{~cm}$ and $y^{\max }=40 \mathrm{~cm}$, calculated as $\left(\Delta_{r e f}^{\max } V-\Delta_{r e f}^{\max } V^{I G}\right) / \Delta_{r e f}^{\max } V^{I G}$ plotted versus the initial reference bubble diameter $d^{r e f}$. The filled markers correspond to the bubbles described in figure 6 .

Figure 7 gives an overview of the deviations for a larger set of bubbles. Two clusters of extreme values can be distinguished. On the one hand, for bubbles with an intermediate size of about $8 \mathrm{~mm}$, significant positive deviations are observed. A representative case is depicted in figure 4 (e), with the corresponding volume evolution plotted in figure 6 for $d^{\text {ref }}=$ $8.4 \mathrm{~mm}$. The deviation between measured and predicted bubble volume increases in a gradual way, giving no direct indication on the underlying cause. On the other hand, for some small bubbles with initial diameters between $5 \mathrm{~mm}$ and $6 \mathrm{~mm}$, a large negative deviation is observed. In this case, a closer look at the deviations reveals that these always coincide with a change in bubble regime. This is clearly illustrated in figure 8 , showing a case in which two regime transitions occur. After injection, the bubble initially follows a periodic regime. At a 
height of about $12 \mathrm{~cm}$, a first transition to a linear regime can be observed, after which the path remains straight for about $10 \mathrm{~cm}$. At this point a second transition occurs, back to a periodic regime. From the bubble volume evolution plotted in figure 8 (c), it is clear that the first regime transitions correlates with the onset of an increasing deviation from the predicted volume, while the opposite trend is observed during the second transition.

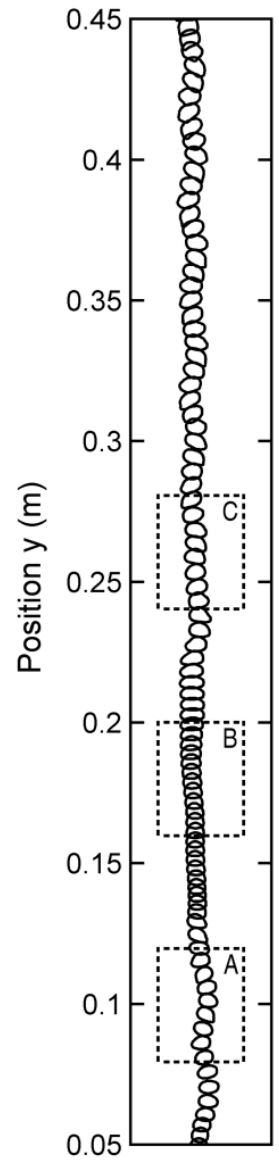

(a)

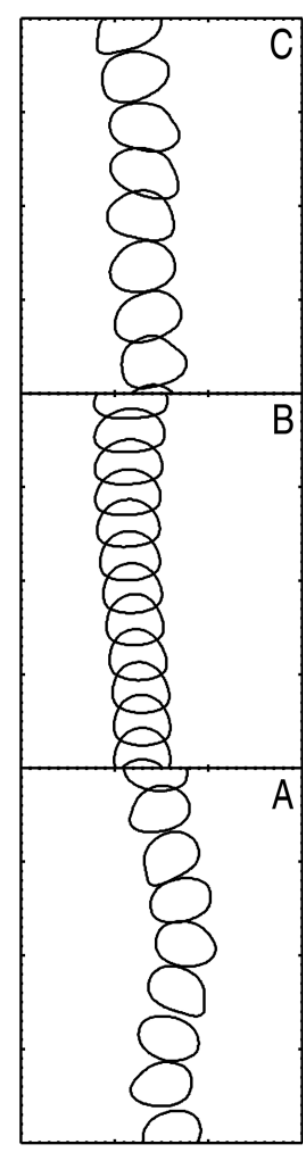

(b)
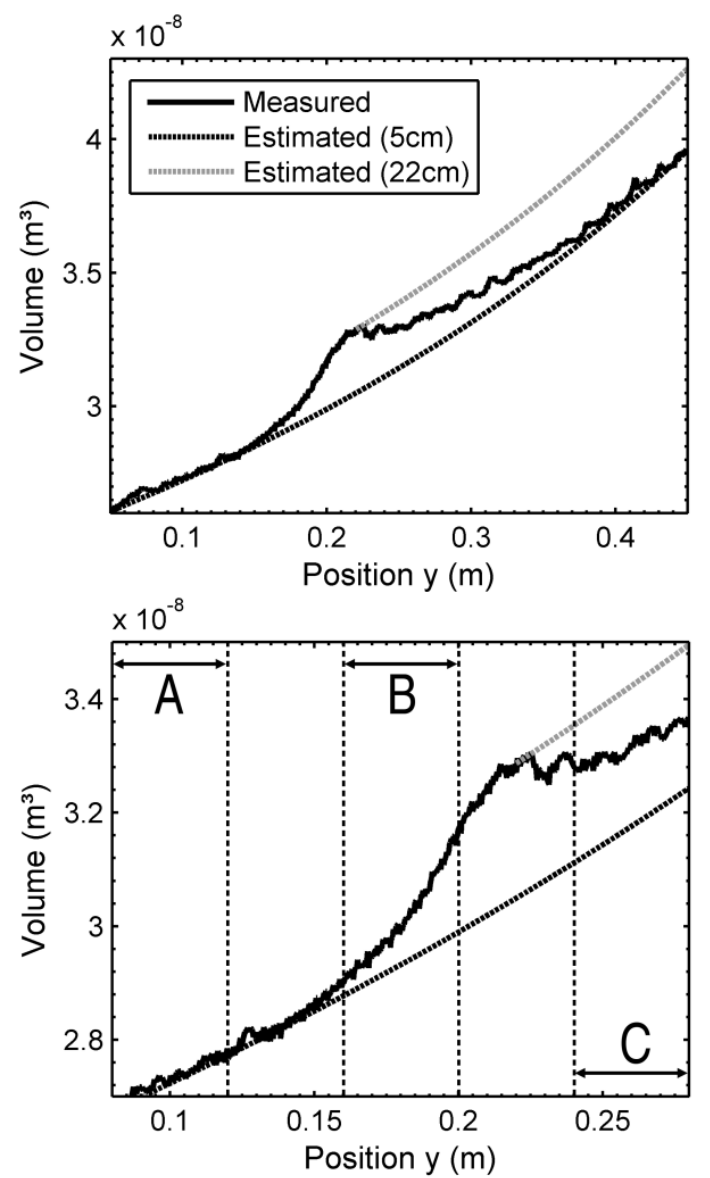

(c)

Fig. 8: (a) Bubble of initial reference diameter of $5.5 \mathrm{~mm}$ showing a transition from a periodic to a linear regime after injection, followed by another transition back to the periodic regime. (b) Close up of the bubble in the three regimes, corresponding to the markers in a. (c) The evolution of the measured bubble volume and predicted volume using $y^{\text {ref }}=$ $5 \mathrm{~cm}$ and $y^{r e f}=22 \mathrm{~cm}$, with a close up of the transition region.

\section{DISCUSSION}

When considering the deviations plotted in figure $7,80 \%$ of their absolute values is below 10 $\%$ and the median value equals $4.3 \%$. This points at a relatively close correspondence between the predicted and observed bubble growth, indicating that all major effects driving the growth of the bubbles are captured by equation (5), using equations (6) and (7) to determine the bubble volume from the recordings. Nevertheless, the larger deviations observed for smaller and intermediate sized bubbles require more explanation. Two scenarios are possible: either the description provided by equation (5) is incomplete, or experimental errors result in an incorrect determination of the observed bubble volume or position. Both scenarios were investigated in order to assess the accuracy of the setup and to understand the behavior of the bubbles. 


\subsection{EXPERIMENTAL ERRORS}

An inaccurate determination of the position of bubbles could be considered as a first source of errors. As mentioned in the setup description, the vertical position of the camera is measured from a ruler pattern on the cell. Because of the way this information is processed, the uncertainty on the position of the camera never exceeds the $4 \mathrm{~mm}$ interval between consequent markers. Compared to this, the uncertainty on the position of the bubble relative to the camera can be neglected considering the resolution of $125 \mu \mathrm{m} / \mathrm{px}$. Furthermore, according to equation (1), a variation of $4 \mathrm{~mm}$ in the vertical position corresponds to a hydrostatic pressure difference of ca. $500 \mathrm{~Pa}$, which is negligible with respect to the total pressure. Hence the errors originating from the position determination cannot explain the deviations in bubble volume.

Secondly, errors could be induced during the determination of the bubble geometry. These errors can be considered by looking at the parameters in equation (6). A first parameter is the cell thickness $t$, for which the standard deviation was measured directly to be $18 \mu \mathrm{m}$. An exact quantification of the spatial error on the position of the bubble contours is less straightforward. However, as can be seen in figure 3, the gas-liquid interface is marked by a sharp spatial gradient in contrast. This indicates that the temporal resolution of the camera, with a shutter time of $2 \mathrm{~ms}$, is sufficient to capture the bubble motion without blurring the images and hence not limiting the spatial resolution. More specifically, the contour gradient spans maximally 3 pixels on the frames recorded by the camera. During processing, the interface is assumed to be halfway this span. Considering the resolution of the camera, for a small circular bubble the standard deviation on the diameter $d$ will thus not exceed $1.5 \mathrm{px}$ or $188 \mu \mathrm{m}$ and the error on the corresponding perimeter $p$ will be below $589 \mu \mathrm{m}$. Assuming a constant contact angle and no correlation between the observed bubble diameter and the cell thickness, the propagation of these errors on $t, d$ and $p$ in the calculation of the bubble volume can be calculated. For a bubble with equivalent diameter of $4 \mathrm{~mm}$, this results in a standard deviation of $9.48 \times 10^{-10} \mathrm{~m}^{3}$ on a bubble volume of $1.40 \times 10^{-8} \mathrm{~m}^{3}$, or a relative error of $6.8 \%$. For larger bubbles the error will be smaller because of the larger volume to interface ratio. Hence this error can partially account for the deviations plotted in figure 7 .

Thirdly, an inaccurate description of the shape of the gas-liquid interface over the cell gap can induce errors on the bubble volume through the parameter $S$ in equation (6). As shown by equation (7), this parameter is directly related to the contact angle $\vartheta_{\mathrm{Hg}}$. Because of the bubble motion, this angle will vary along the nitrogen-mercury-glass triple lines (de Gennes et al. 2003). In the past, experimental efforts have been undertaken to measure the dynamic contact angles for the particular case of mercury droplets on glass, used in porosimetry (Salmas and Androutsopoulos 2001). For flow in submicron pores, receding contact angles are found to be about $104^{\circ}$ while advancing angles are equal to $140^{\circ}$. It is however not clear to what extent these values can be extrapolated to the current, macroscopic system. In addition, the authors are not aware of a model that can accurately predict the variation of the angle from the position on the contact line. Hence a constant contact angle of $130^{\circ}$ is assumed here, equal to the static contact angle. This seems reasonable because the triple lines on both walls form close loops on which the positive difference for the advancing part of the line will partly cancel out the effect of the smaller angle at the receding part. 
Nevertheless, the assumption will introduce an error when determining the volume of a bubble according to equations (6) and (7). To estimate this effect, the relative change in bubble volume upon selecting a different contact angle is plotted in figure 9 . This shows that in the case of perfect dewetting, when $\vartheta_{H g}$ would be $180^{\circ}$, even for the smallest bubbles the deviation is still limited to $5 \%$. In the other extreme case of $\vartheta_{H g}=90^{\circ}$, with $S$ being zero according to equation (7), the bubble volume for a $4 \mathrm{~mm}$ diameter bubble would be $6 \%$ smaller compared to the $\vartheta_{H g}=130^{\circ}$ case. Notice that for angles below $90^{\circ}$, corresponding to the highly unlikely case in which mercury would wet the glass, the deviation would also be between these two extremes. The reason is that the curvature of the interface depicted in figure 5 would change from convex to concave and the expression for $S$ should be adapted accordingly.

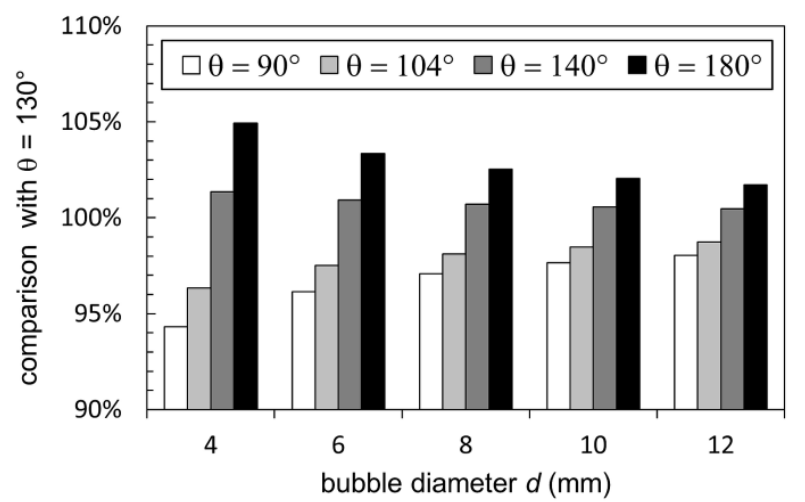

Fig. 9: A plot of the bubble volume for different diameters $d$ and contact angles $\vartheta_{H g}$, compared to the case in which $\vartheta_{H g}$ equals $130^{\circ}$.

To summarize it can be stated that the experimental uncertainty on the results is caused mainly by two factors. Firstly there is the limited spatial resolution of the observations, caused by both limitations on the camera and the construction of the setup. From this point of view, the quality of the experiments could be increased by using a camera with better specifications and a cell with lower dimensional tolerances. Secondly there is the limited knowledge on the exact shape of the gas-liquid interface. A first requirement to overcome this limitation would be the development of a detailed macroscopic description of the contact line dynamics near the cell walls. This is however outside the scope of this work. The two factors make about an equal contribution, both decreasing with increasing bubble size. Together these probably account for the majority of deviations shown in figure 7. However, the large negative deviations for smaller bubbles, up to $25 \%$, cannot be explained in this way, suggesting that the description provided by equation (5) is incomplete.

\subsection{VOLUME EFFECT OF REGIME TRANSITIONS}

As mentioned before, the deviations observed for smaller bubbles seem to be correlated with regime transitions. An example is given in figure 8 . This is a special case because it shows two consecutive regime transitions whereas only the second linear to periodic transition would be expected for a small bubble starting to deform and oscillate with increasing size (Clift et al. 1978). In this case the first periodic to linear transition is probably caused by transient effects related to the injection of the bubble. It seems plausible that kinetic energy supplied to the system during the injection of a burst of gas could temporarily support the 
initial periodic behavior of the flow. This would explain a transition towards the more stable, linear regime after dissipation of this excess energy. The bubble then continues along a straight path until its increasing size justifies the second transition, back to the periodic regime. Figure 8 (c) shows that the observed bubble size during this linear regime seems to be relatively larger compared to the periodic regime. This suggests that a more accurate prediction of the bubble size evolution should also account for the effect of changing flow dynamics between the different flow regimes. Hence, equation (5) can only be used to predict the bubble size evolution when the regime at the reference point $y^{\text {ref }}$ is similar to that at $y^{\max }$. This explains the large negative deviations in figure 7 for small bubbles with an initial diameter between $5 \mathrm{~mm}$ and $6 \mathrm{~mm}$, near the expected transition size. When a linear to periodic transition occurs between $y^{\text {ref }}$ and $y^{\max }$, it is likely that the bubble volume is overestimated, as demonstrated in figure 8 (c), where predictions based on both $y^{\text {ref }}=5 \mathrm{~cm}$ and $y^{r e f}=22 \mathrm{~cm}$ are plotted. In the first case, with $y^{r e f}$ and $y^{\max }$ both situated in a periodic regime, the resulting error at $y^{\max }$ is near zero while in the second case, with $y^{\text {ref }}$ in the linear regime, the error is significantly larger.

Because the volume effect is significant with respect to the experimental accuracy, and because it is flow dependent, it can only be explained by a variation of flow induced dynamic pressure inside the bubble. Hence, in order to predict the expansion of a bubble accurately, a dynamic pressure term $P_{d y n}$ should be added in addition to the static pressure components already included in equation (2):

$$
P(d, y)=P_{a t m}+P_{h y d}(y)+P_{c a p}(d)+P_{d y n}
$$

Furthermore, in figure 8 it can be seen that, despite the fact that the initial periodic behavior at $y^{r e f}=5 \mathrm{~cm}$ is unstable, it provides a good reference to predict the bubble size at $y^{\text {max }}=$ 40 in a similar periodic regime. This means that the volume effect of a periodic to linear transition is exactly opposing the effect of the inverse, linear to periodic transition, suggesting that $P_{d y n}$ is mainly regime dependent. From a comparison between the measured and predicted volumes, it can be concluded that $P_{d y n}$ will be smaller for the linear bubble regime compared to the periodic regime. This seems logical, considering that flow induced dynamic pressure components in low Reynolds number systems can only originate from the same effects that cause the swirling of the bubble and that are hence more important in periodic regimes.

Due to their expansion, small bubbles with an initial size below $6 \mathrm{~mm}$ typically change from a linear regime to a periodic regime somewhere along their path. Hence, if this transition happens between a reference position at $y^{\text {ref }}=15 \mathrm{~cm}$ and a position at $y^{\max }=30 \mathrm{~cm}$, $P_{d y n}$ will increase stepwise and partly compensate the hydrostatic pressure decrease, resulting in a smaller than expected bubble growth. This explains the significant deviations for small bubbles in figure 7 . Finally it can be mentioned that an extrapolation of this scenario could also explain the larger positive deviations for bubble sizes around $8 \mathrm{~mm}$. When comparing the $8.7 \mathrm{~mm}$ bubble with the $7.0 \mathrm{~mm}$ bubble in figure 4, it is clear that the amplitude of the swirling motion is smaller for the larger bubble, despite the fact that both contain some periodicity in their shape. So irrespective of whether the regime for these 
bubbles is different, it can be stated that the motion of the larger bubble resembles more to that of a bubble in the linear regime. Hence, if the dynamic pressure difference changes accordingly during the rise of an intermediate bubble, a faster than predicted growth can be expected in this range of bubble diameters. This could lead to the positive deviations in figure 7. In addition, a comparison of the path for bubbles near this size shows that the swirling motion reduces gradually with increasing size. This could explain why the deviation for intermediate bubbles increase gradually, as observed in figure 6, while the deviations for smaller bubbles develop rather stepwise, together with the regime transition.

\section{CONCLUSION}

In this article, the use of a Hele-Shaw cell is proposed to observe gas bubbles in liquid metal at a mesoscopic scale with a high resolution. The flow in such a cell can be fully mapped by two dimensional observations, avoiding the need for tomography. Under the right circumstances, a direct visualization of the bubbles through transparent cell walls is possible, although the geometry of the setup facilitates the application of all types of hard field tomography. A case study on buoyancy driven nitrogen bubbles in liquid mercury in a transparent cell demonstrates the viability of the proposed technique. By using a high speed camera that travels upward with the rising bubbles, clear and sharp close up recordings along their entire path could be made. This would not be possible if a fixed camera had been used. The resolution of the resulting images far exceeds results reported elsewhere in literature and obtained with alternative techniques. This revealed the existence of two flow regimes within the range of bubble diameters between $4 \mathrm{~mm}$ and $12 \mathrm{~mm}$ : smaller bubbles exhibit a linear regime with an elliptical shape and a straight path, while larger bubbles move along a swirling path and show a periodically distorted shape.

Furthermore, it is demonstrated how the observations are translated into quantitative descriptions of the bubble properties, including a detailed assessment of the resulting experimental errors. As a practical example of the applicability of these descriptions, the evolution of the bubble volume along the path is analyzed. It is demonstrated how the hydrostatic pressure gradient over the cell height causes a significant expansion of the bubbles. This phenomenon is of particular importance for the case of liquid metals with a typically high density, and is therefore difficult to demonstrate with more commonly studied aqueous systems. In addition it is shown that the volume effect of a changing dynamic pressure is also significant and largely regime dependent. It can also be mentioned that the authors plan to provide a more detailed description of the bubble properties, together with a more elaborate discussion on the underlying flow physics, in a subsequent publication.

It is however clear that, due to the two-dimensional nature of the flow, extrapolations to more practically relevant conditions are not straightforward. From this point of view, a Hele-Shaw based approach is complimentary to experimental techniques that allow three dimensional observations. Nevertheless, the authors believe that the suggested technique offers a fast and simple way to gather additional data on the mesoscopic interactions between microscopic and macroscopic phenomena. Hence it can be considered as a first, easily accessible step towards a full experimental study of the gas liquid interactions in various metallurgical systems. 


\section{ACKNOWLEDGMENTS}

This research was supported by the Agency for Innovation by Science and Technology in Flanders and by Umicore Group Research \& Development (IWT Baekeland mandate 090271).

\section{REFERENCES}

Baker MC, Bonazza R (1998) Visualization and measurements of void fraction in a gasmolten tin multiphase system by X-ray absorption. Exp Fluids 25 (1):61-68. doi: $10.1007 / \mathrm{s} 003480050208$

Bauer M, Eigenberger G (2001) Multiscale modeling of hydrodynamics, mass transfer and reaction in bubble column reactors. Chem Eng Sci 56 (3):1067-1074. doi:10.1016/S0009-2509(00)00323-7

Beavers GS, Sparrow EM, Magnuson RA (1970) Experiments on breakdown of laminar flow in a parallel-plate channel. Int J Heat Mass Tran 13 (5):809-815. doi:10.1016/00179310(70)90127-4

Bouche E, Roig V, Risso F, Billet AM (2012) Homogeneous swarm of high-Reynolds-number bubbles rising within a thin gap. Part 1. Bubble dynamics. J Fluid Mech 704:211-231. doi:10.1017/Jfm.2012.233

Boyer C, Duquenne AM, Wild G (2002) Measuring techniques in gas-liquid and gas-liquidsolid reactors. Chem Eng Sci 57 (16):3185-3215. doi:10.1016/S0009-2509(02)001938

Bush JWM (1997) The anomalous wake accompanying bubbles rising in a thin gap: a mechanically forced Marangoni flow. J Fluid Mech 352:283-303. doi:10.1017/S0022112097007350

Bush JWM, Eames I (1998) Fluid displacement by high Reynolds number bubble motion in a thin gap. Int J Multiphas Flow 24 (3):411-430. doi:10.1016/S0301-9322(97)00068-2

Clift R, Grace JR, Weber ME (1978) Bubbles, drops, and particles. Academic Press, London

de Gennes PG, Brochard-Wyart F, Quere D (2003) Capillarity and wetting phenomena: drops, bubbles, pearls, waves. Springer, New York

Eck W, Siekmann J (1978) Bubble motion in a Hele-Shaw cell, a possibility to study 2-phase flows under reduced gravity. Ing Arch 47 (3):153-168

Engh TA, Simensen CJ, Wijk O (1992) Principles of metal refining. Oxford Unversity Press, Oxford, UK

Fu QA, Evans JW (1999) A capacitance probe for measurement of bubbles in molten metals. Isij Int 39 (4):309-318. doi:10.2355/isijinternational.39.309

Grechko AV (2000) Prospects for the use of bubbling processes in pyrometallurgy. Metallurgist+ 44 (3-4):151-154. doi:10.1007/Bf02466165

He YQ, Bi QC, Shi DX (2011) Dynamics of a single air bubble rising in a thin gap filled with magnetic fluids. Fluid Dyn Mater Process 7 (4):357-370

Heindel TJ (2011) A review of X-ray flow visualization with applications to multiphase flows. J Fluid Eng-T Asme 133 (7). doi:10.1115/1.4004367

Huisman SG, Ern P, Roig V (2012) Interaction and coalescence of large bubbles rising in a thin gap. Phys Rev E 85 (2). doi:10.1103/Physreve.85.027302 
Iguchi M, Chihara T, Takanashi N, Ogawa Y, Tokumitsu N, Morita Z (1995) X-ray fluoroscopic observation of bubble characteristics in a molten iron bath. Isij Int 35 (11):1354-1361. doi:10.2355/isijinternational.35.1354

Iguchi M, Ilegbusi OJ (2011) Modeling multiphase materialsprocesses: gas-liquid systems Springer, New York

Iguchi M, Nakatani T, Kawabata H (1997) Development of a multineedle electroresistivity probe for measuring bubble characteristics in molten metal baths. Metall Mater Trans B 28 (3):409-416. doi:10.1007/s11663-997-0106-3

Jakobsen HA, Lindborg H, Dorao CA (2005) Modeling of bubble column reactors: progress and limitations. Ind Eng Chem Res 44 (14):5107-5151. doi:10.1021/le049447x

Johann W, Siekmann J (1978) Migration of a bubble with adsorbed film in a Hele-Shaw cell. Acta Astronaut 5 (9):687-704. doi:10.1016/0094-5765(78)90048-6

Kawaguchi M, Niga S, Gou N, Miyake K (2006) Buoyancy-driven path instabilities of bubble rising in simple and polymer solutions of Hele-Shaw cell. J Phys Soc Jpn 75 (12). doi:10.1143/Jpsj.75.124401

Kelley E, Wu MM (1997) Path instabilities of rising air bubbles in a Hele-Shaw cell. Phys Rev Lett 79 (7):1265-1268. doi:10.1103/PhysRevLett.79.1265

Kopf-Sill AR, Homsy GM (1988) Bubble motion in a Hele-Shaw cell. Phys Fluids 31 (1):1826. doi:10.1063/1.866566

Kozuka H, Ikeda Y, Kawaguchi M (2009) Path instabilities of bubble rising in polymer solutions of Hele-Shaw cell. J Phys Soc Jpn 78 (11). doi:10.1143/Jpsj.78.114604

Marashdeh Q, Fan LS, Du B, Warsito W (2008) Electrical capacitance tomography - a perspective. Ind Eng Chem Res 47 (10):3708-3719. doi:10.1021/le0713590

Maxworthy T (1986) Bubble formation, motion and interaction in a Hele-Shaw cell. J Fluid Mech 173:95-114. doi:10.1017/S002211208600109x

Mazumdar D, Evans JW (2004) Macroscopic models for gas stirred ladles. Isij Int 44 (3):447461. doi:10.2355/isijinternational.44.447

Munshi P, Jayakumar P, Satyamurthy P, Thiyagarajan TK, Dixit NS, Venkatramani N (1998) Void-fraction measurements in a steady-state mercury-nitrogen flow loop. Exp Fluids 24 (5-6):424-430. doi:10.1007/s003480050192

Oryall GN, Brimacombe JK (1976) Physical behavior of a gas-jet injected horizontally into liquid-metal. Metall Trans B 7 (3):391-403. doi:10.1007/Bf02652710

Paneni M, Davenport WG (1969) Dynamics of bubbles in liquid metals - two-dimensional experiments. T Metall Soc Aime 245 (4):735-738

Park CW, Maruvada SRK, Yoon DY (1994) The influence of surfactant on the bubble motion in Hele-Shaw cells. Phys Fluids 6 (10):3267-3275. doi:10.1063/1.868059

Roig V, Roudet M, Risso F, Billet AM (2012) Dynamics of a high-Reynolds-number bubble rising within a thin gap. J Fluid Mech 707:444-466. doi:10.1017/Jfm.2012.289

Saito Y, Mishima K, Tobita Y, Suzuki T, Matsubayashi M, Lim IC, Cha JE (2005) Application of high frame-rate neutron radiography to liquid-metal two-phase flow research. Nucl Instrum Meth A 542 (1-3):168-174. doi:10.1016/j.nima.2005.01.095

Saito Y, Shen X, Mishima K, Matsubayashi M (2009) Shape measurement of bubble in a liquid metal. Nucl Instrum Meth A 605 (1-2):192-196. doi:10.1016/j.nima.2009.01.168

Salmas C, Androutsopoulos G (2001) Mercury porosimetry: contact angle hysteresis of materials with controlled pore structure. J Colloid Interf Sci 239 (1):178-189. doi:10.1006/jcis.2001.7531 
Santos LMT, Esteves MTMS, Pinheiro MNC (2008) Effect of gas expansion on the velocity of individual Taylor bubbles rising in vertical columns with water: Experimental studies at atmospheric pressure and under vacuum. Chem Eng Sci 63 (18):4464-4474. doi:10.1016/j.ces.2008.06.018

Schneider M, Evans JW (2006) A novel probe for detecting gas bubbles in liquid metals. Metall Mater Trans B 37 (3):333-337. doi:10.1007/s11663-006-0018-7

Schwerdtfeger K (1968) Velocity of rise of argon bubbles in mercury. Chem Eng Sci 23 (8):937-938. doi:10.1016/0009-2509(68)80030-2

Smithwick RW (1988) Contact-angle studies of microscopic mercury droplets on glass. J Colloid Interf Sci 123 (2):482-485. doi:10.1016/0021-9797(88)90269-X

Span R, Lemmon EW, Jacobsen RT, Wagner W, Yokozeki A (2000) A reference equation of state for the thermodynamic properties of nitrogen for temperatures from 63.151 to $1000 \mathrm{~K}$ and pressures to $2200 \mathrm{MPa}$. J Phys Chem Ref Data 29 (6):1361-1433. doi:10.1063/1.1349047

Taylor IF, Wright JK, Gray NB Liquid mixing resulting from reactive gas injection. In: M. N, T. $L$ (eds) The Howard Worner International Symposium on Injection in Pyrometallurgy, Melbourne, 1996. Minerals, Metals \& Materials Society pp 163-174

Terzija N, Yin WL, Gerbeth G, Stefani F, Timmel K, Wondrak T, Peyton A (2011) Electromagnetic inspection of a two-phase flow of GalnSn and argon. Flow Meas Instrum 22 (1):10-16. doi:10.1016/j.flowmeasinst.2010.10.003

Thiyagarajan TK, Satyamurthy P, Dixit NS, Venkatramani N, Garg A, Kanvinde NR (1995) Void fraction profile measurements in 2-phase mercury-nitrogen flows using gammaray attenuation method. Exp Therm Fluid Sci 10 (3):347-354. doi:10.1016/08941777(94)00082-J

Vasil'ev A (2009) From the Hele-Shaw experiment to integrable systems: A historical overview. Complex Anal Oper Th 3 (2):551-585. doi:10.1007/s11785-008-0104-8

Versluis M (2013) High-speed imaging in fluids. Exp Fluids 54 (2):1-35. doi:10.1007/s00348013-1458-X

Wang F, Marashdeh Q, Fan LS, Warsito W (2010) Electrical capacitance volume tomography: design and applications. Sensors-Basel 10 (3):1890-1917. doi:10.3390/S100301890

Yamamoto M, Kawaguchi M (2011) Differences in path instabilities between a bubble rising in water and in aqueous polymer solution in a Hele-Shaw cell in the transient and steady states. J Disper Sci Technol 32 (10):1445-1451. doi:10.1080/01932691.2010.513305

Zhang C, Eckert S, Gerbeth G (2007) The flow structure of a bubble-driven liquid-metal jet in a horizontal magnetic field. J Fluid Mech 575:57-82. doi:10.1017/S0022112006004423 